\section{LA CONSTRUCCIÓN DEL ESPACIO EUROPEO DE EDUCACIÓN SUPERIOR Y LA INNOVACIÓN EDUCATIVA EN LA UNIVERSIDAD}

\author{
Jesús Arriaga García de Andoaín \\ y Carlos Conde Lázaro \\ Vicerrectorado de Ordenación Académica y Planificación Estratégica \\ Universidad Politécnica de Madrid
}

\begin{abstract}
In the present article, we put forth a reflection about the roll that the renewal of teaching methods used in the learning process for University Degrees must play, within the European Space for Higher Education framework development. We illustrate this, with the activities carried out at the Universidad Politécnica de Madrid during the last four years.
\end{abstract}

KEY WORDS: Educational Innovation; EEES; quality institutional program; "adjustments" and updating; starting point; groups for the educational innovation.

\section{INTRODUCCIÓN}

Todas las Universidades vivimos actualmente la necesidad de dar respuesta a nuevas necesidades en el ámbito educativo. Esta necesidad es permanente en cualquier Universidad que, como la Universidad Politécnica de Madrid, tiene el compromiso de dar el mejor servicio a la sociedad. Sin embargo, de lo que hoy hablamos no es un simple cambio cuantitativo en este proceso de adaptación, sino de uno de esos cambios cualitativos que cíclicamente se producen en la historia de los fenómenos sociales.

Las razones son bien conocidas y han sido etiquetadas con el nombre de "proceso de Bolonia", aunque, como suele suceder con los temas complejos a los que se encuadran excesivamente, se corre el peligro de perder la perspectiva y profundidad de las razones en las que se sustenta el cambio. Buscamos una Universidad más eficiente, más permeable con la sociedad que le rodea, más proyectada universalmente y que desde el punto de vista formativo de las personas no sea una etapa final sino un eslabón más en un aprendizaje a lo largo de la toda la vida.
THE CONSTRUCTION OF EUROPEAN SPACE FOR HIGHER EDUCATION AND THE EDUCATIVE INNOVATION AT THE UNIVERSITY

RESUMEN: En el presente artículo se realiza una reflexión sobre el papel que debe jugar la renovación de los métodos docentes utilizados en el proceso formativo de titulados universitarios en el marco de la construcción del Espacio Europeo de Educación Superior. Lo anterior se ilustra con las actividades que se han venido desarrollando en la Universidad Politécnica de Madrid durante los últimos cuatro años.

PALABRAS CLAVE: Innovación Educativa; EEES; programa institucional de calidad; puesta a punto; punto de inicio; grupos de innovación educativa.

En líneas generales se puede afirmar que conocemos con suficiente precisión hacia dónde debemos avanzar, pero lo que no está tan claro son los caminos por los que hay que adentrarse. Éste es un problema general de todo cambio educativo porque dichos cambios no son posibles realizarlos sin la corresponsabilidad y complicidad de una amplia mayoría de los profesores y personal de administración y servicios y la aceptación de los estudiantes basada en la identificación son sus objetivos.

La dificultad aludida como inherente al cambio educativo, también se produce en todos los niveles de la enseñan$z a$, si bien en la Universidad presenta muchos aspectos singulares que deben enmarcarse en la autonomía de las

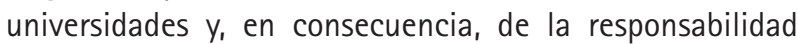
que deben asumir.

En este contexto, la renovación docente de las enseñanzas universitarias se ha convertido en una línea estratégica de actuación de todas las universidades. Para orientarla se han generado marcos de referencia a través de los cuales se están desarrollando iniciativas que dinamicen 
e impulsen a mayor escala lo que ha sido una actividad permanente, pero de baja intensidad, en el sistema universitario español.

La Universidad Politécnica de Madrid, no ha sido una Universidad que tradicionalmente haya estado muy presente en los foros educativos. Ello se debió no tanto a la falta de interés y ejercicio de la innovación educativa sino a la la débil estructuración de estas actividades y la escasa atención por su proyección. Sin embargo, la situación en los últimos cinco años ha cambiado sustancialmente desarrollándose una política activa de innovación educativa en torno a tres grandes marcos de referencia.

\section{Marcos de referencia de la inNovación educativa en la Universidad Politécnica}

1. El Programa Institucional de la Calidad en la UPM, como principal marco de referencia para la Innovación Educativa, define un "Plan General de calidad de la Enseñanza" que a su vez se desglosa en dos "Proyectos", denominados Proyecto Convergencia al EEES y Proyecto Calidad de la Oferta Formativa-UPM. En ellos se describen diferentes actuaciones, entre las que destaca la convocatoria de Proyectos de Innovación Educativa, y estable pautas y objetivos que posteriormente han sido desarrolladas en las diferentes convocatorias de proyectos.

Con criterio general se establecen los siguientes objetivos generales para las actuaciones de la Innovación Educativa:

- Adaptación al Espacio Europeo de Educación Superior, entendiendo esta adaptación de acuerdo a los siguientes principios:

$\checkmark$ Establecimiento de un sistema de créditos transferible y cambios metodológicos que lo posibiliten.

$\checkmark$ Promoción de la movilidad de estudiantes y profesores.

$\checkmark$ Reorganización de la estructura de títulos de acuerdo a las singularidades de la Universidad Politécnica de Madrid.

$\checkmark$ Establecimiento de sistemas de "garantía de calidad".
- Mejora de la eficiencia de los programas formativos impartidos en la UPM. Se pretende mejorar en algunos de los puntos débiles generales de la Universidad Politécnica de Madrid (tasa de "abandono", duración de los estudios,...) sin que eso suponga una pérdida de posición en otros de los puntos fuertes de nuestra Universidad (formación de nuestros ingenieros, prestigio en la empresa, excelente inserción profesional de los egresados,....)

- Incorporación de objetivos formativos competenciales en la enseñanza reglada. Para conducir el proceso anterior, la Universidad Politécnica de Madrid ha desarrollado diferentes estudios sobre las principales características que deben tener los profesionales formados en sus aulas. En las consultas realizadas sobre la formación de grado en la UPM, dirigidas tanto a representantes empresariales como a los egresados de distintas promociones, hay plena coincidencia en señalar su alta cualificación profesional pero también muestran algunos puntos de mejora altamente demandados. El Gráfico 1 recoge un resumen de la cobertura de ciertas competencias (en una escala de 1 a 10) en relación con las necesidades concretas que los egresados han necesitado o consideran que van a necesitar para el desempeño de su ejercicio profesional.

En consecuencia con estos análisis, se han definido ocho competencias nucleares que deben ser incorporadas en todos los nuevos programas formativos impartidos en la UPM. Éstas han quedado formuladas para las titulaciones de Grado de la siguiente manera:

- Uso de la lengua inglesa

- Trabajo en equipo

- Comunicación oral y escrita

- Uso de Tecnologías de la Información y de las Comunicaciones

- Creatividad.

- Liderazgo de equipos

- Organización y planificación

- Capacidad para el diseño sostenible

- Participación activa de los estudiantes en los procesos educativos, asumiendo su parte de responsabilidad no sólo en los procesos de aprendizaje, sino en las dinámicas innovadoras que se pretenden relanzar. 
Gráfico 1. Cobertura del nivel de competencias en relación con las necesidades concretas que han tenido o consideran que van a necesitar para el desempeño de su ejercicio profesional.

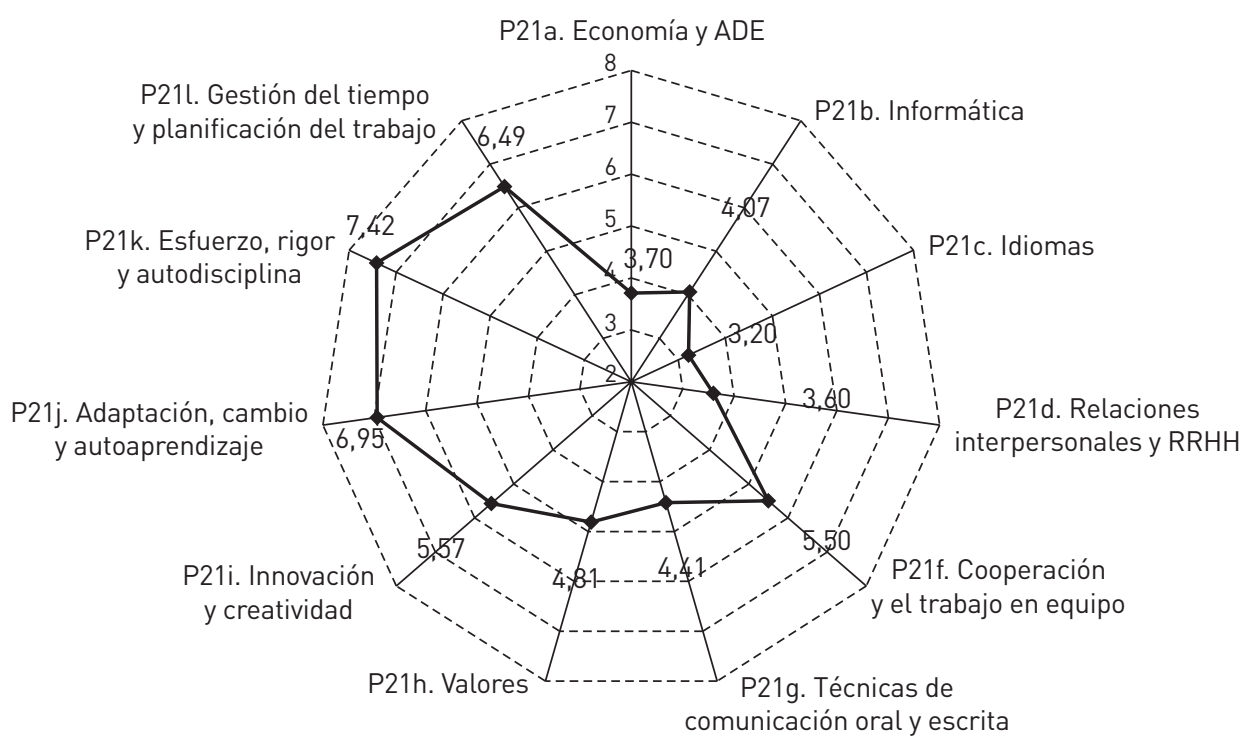

- Desarrollo de actuaciones contempladas en los Programas de Mejora que se deriven de Planes de Evaluación Institucional o, en su caso, de Planes definidos por los Consejos de Departamento, la Junta de Centro o el Rectorado.

2. La regulación del proceso de elaboración de los nuevos programas formativos es, sin duda, el segundo gran marco de referencia tanto en lo que se refiere a las directrices generales como a los requisitos y recomendaciones de la propia Universidad.

El Real Decreto 1393/2007, de 29 de octubre, por el que se establece la ordenación de las enseñanzas universitarias oficiales ha resumido muchos trabajos anteriores y ha supuesto un cambio profundo en el planteamiento de concebir y diseñar los nuevos programas formativos de la educación superior. La síntesis de este cambio cultural se refleja en una nueva forma de entender los Planes de Estudio como un contrato entre la Universidad, los estudiantes y la sociedad y cuyos puntos a desarrollar se resumen en los diez conocidos apartados:

1. Descripción del título.

2. Justificación.
3. Objetivos.

4. Admisión de estudiantes.

5. Planificación de la enseñanza.

6. Personal académico.

7. Recursos materiales y servicios.

8. Resultados previstos.

9. Sistemas de garantía de calidad.

10. Calendario de implantación.

Todos ellos, colectivamente, constituyen una unidad que dan sentido al proyecto y permiten planificar de forma integrada y coherente los nuevos Planes de Estudio. Sin embargo, y reconociendo que la importancia de cada elemento sólo se puede valorar dentro del conjunto, cabe destacar dos elementos especialmente novedosos:

a) La Definición de Objetivos y la Planificación de la enseñanza. Los nuevos planes de estudios deben incluir una descripción de las competencias transversales y específicas a adquirir por el estudiante que deben haber adquirido los estudiantes durante sus estudios y que sean exigibles para otorgar el título. Todas las acciones curriculares habrán de estar dirigidas a que los estudiantes adquieran dichas competencias $y$, por lo tanto, deberán trasladarse al plan de estudios. 
En él podrán adoptarse diferentes estructuras para describir un plan de estudios, desde la tradicional estructura de asignaturas con reducido número de créditos -con resultado de atomización del plan de estudios-, pasando por estructuras más globales, módulos y/o materias, que permitan una organización flexible y capaz de responder con mayor eficacia a logros de los objetivos de formación previstos.

La coherencia entre los objetivos y la estructura de las enseñanzas debe estar justificada mediante la interrelación de ambas expresada en términos de competencias y resultados de aprendizaje que el estudiante adquiere en cada uno de los módulos o materias del Plan de Estudios.

b) Sistemas de Garantía de Calidad y resultados previstos. Éstos podrán referirse tanto a un sistema propio de la titulación como a un sistema general de la universidad o del centro responsable de las enseñanzas, aplicable a la titulación, pero en cualquiera de los casos se deberá garantizar que se dispone de un sistema de autocontrol y mejora de la planificación establecida. Especial mención debe hacerse de la importancia dada a los resultados de eficiencia académica, medidos a través de tres tasas: Tasa de Graduación. Tasa de Abandono y Tasa de Eficiencia. La eficacia y la eficiencia del sistema universitario español es uno de los puntos sobre los que específicamente se demanda un análisis y en muchos casos una mejora significativa.

3. El tercer marco de referencia para la Innovación Educativa lo constituyen los "Acuerdos-Programa para la mejora de la calidad de los centros universitarios" que permitirán, conocidas las necesidades de mejora, las posibilidades de actuación y los datos de partida de cada Centro, establecer objetivos concretos de mejora para cada uno de ellos, en un período máximo de cuatro años, y con un seguimiento, al menos anual, en torno a las siguientes finalidades:

- Adaptar la oferta formativa a las necesidades sociales y del tejido empresarial.

- Contribuir a la adaptación de las metodologías docentes a las directrices del Espacio Europeo de Educación Superior.

- Incrementar la eficiencia y la calidad de los procesos formativos.
- Dotar de una dimensión internacional a nuestras enseñanzas.

- Diversificar y distribuir la formación a lo largo de la vida.

- Hacer realidad la conexión entre el I+D+i, la transferencia de resultados y la docencia.

- Potenciar el uso de las TIC en los procesos formativos.

- Difundir nacional e internacionalmente la imagen UPM a través de cada uno de sus Centros, e informar sobre la contribución de la universidad a la sociedad.

- Introducir criterios de Distribución de recursos acordes con los objetivos logrados.

- Estimular la mejora continua de los Centros de la UPM.

El Acuerdo-Programa se establece en tres líneas de actuación:

- LÍNEA 1: De distribución de la asignación presupuestaria de gastos corrientes, tendente a ajustar la asignación presupuestaria del gasto corriente de cada Centro en función de su actividad real,

- LíNEA 2: De apoyo a la implementación de los Planes de Mejora, tendente a propiciar la implantación de acciones de mejora orientada a la consecución, a medio y largo plazo, de objetivos institucionales de la universidad, y

- LíNEA 3: De mejora continua de los Centros, tendente a mejorar a corto y medio plazo algunos indicadores de actividad.

A través de los objetivos marcados en las tres líneas de trabajo se desgrana un conjunto de actuaciones para cada Centro en función de las necesidades de mejora, de las posibilidades de actuación y de los datos de partida. Sin ánimo de hacer una relación exhaustiva de los objetivos relacionados con la Innovación Educativa se pueden destacar:

Línea 1: De distribución de la asignación presupuestaria de gastos corrientes:

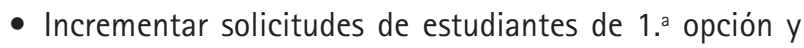
de nuevo ingreso.

- Mejorar la eficiencia del proceso formativo.

- Incrementar formación y experiencias de Innovación Educativa.

- Incrementar acciones de movilidad. 
Línea 2: De apoyo a la implantación de planes de mejora:

- Planificación del Programa formativo.

- Proceso de Enseñanza-Aprendizaje y evaluación.

- Acompañamiento y comunicación con los alumnos

- Formación y ayudas al profesorado y PAS

Línea 3: De mejora continua de los Centros:

- Actividad educativa referida particularmente a:

- Aumentar la demanda y matrícula de los estudios de grado del Centro en número, procedencia y nota de acceso.

- Mejorar el flujo de los estudiantes de grado incrementando el número de titulados, asegurando que los contenidos formativos y su carga docente se ajusten a los requisitos de calidad de la enseñanza.

- Mejorar la Internacionalización de los estudios del Centro.

- Adaptar los estudios del Centro a las directrices del EEES potenciando el papel activo del alumno y la utilización de las TIC como estrategia de mejora del aprendizaje y rendimiento académico.
- Conocer y mejorar la inserción laboral de los titulados utilizando la información resultante para la mejora de la adecuación del perfil de egreso a las demandas sociales.

- Fidelización de antiguos alumnos

Varias son las reflexiones que se pueden realizar a partir de los enunciados anteriores, pero en estos momentos conviene hacer una referencia concreta a la preocupación de la Universidad Politécnica de Madrid por la caída en el interés de los jóvenes por los estudios científicotecnológicos.

En el Gráfico 2 se muestra la evolución del número de estudiantes matriculados en las PAU por la opción de Ciencia y Tecnología en Madrid y en España. Estos datos muestran una tendencia divergente de los objetivos definidos en la Declaración de Lisboa en la que se reconoce el interés estratégico para los países de los estudios científico-tecnológicos y con los que la Universidad Politécnica de Madrid se siente especialmente comprometida. De ahí el interés de fomentar las vocaciones y el interés de los estudiantes de enseñanzas medias por este tipo de estudios.

Gráfico 2. Evolución del número de estudiantes matriculados en las PAU por la opción de Ciencia y Tecnología en Madrid y en España.

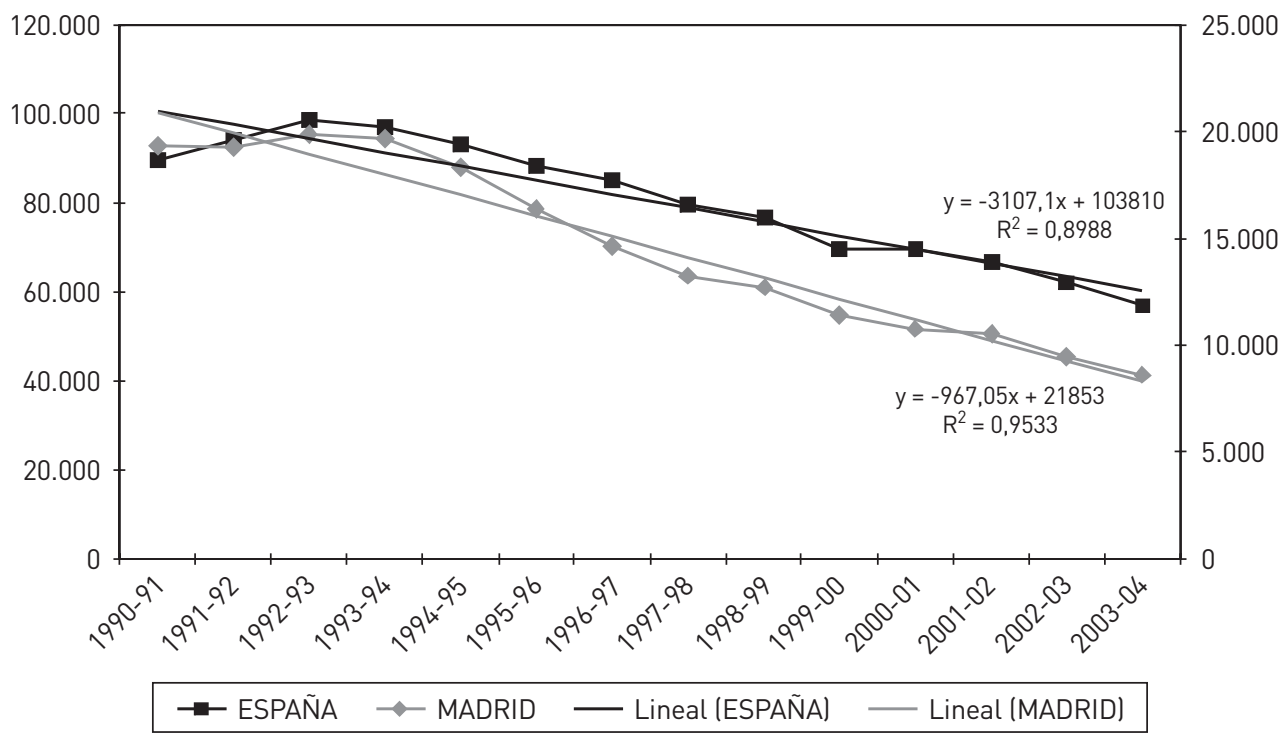


En los marcos de referencia descritos anteriormente existen, como no podía ser de otra manera, una gran coincidencia en las líneas de actuación en las que la UPM considera prioritario desarrollar la Innovación Educativa. En todas ellas está presente la integración en el Espacio Europeo de Educación Superior como el principal motivo de reforma y oportunidad de mejora.

\section{InStRumentos E InICIATIVAS PARA EL DESARROLlO de la InNovación Educativa en la Universidad}

Definidos los marcos de referencia, se han desarrollado una variedad de instrumentos que permitieran impulsar la participación de la comunidad universitaria en la consecución de los objetivos marcados, proporcionando los medios y estimulando su motivación intrínseca y extrínseca. Los más relevantes han sido los siguientes:

- Los Grupos de Innovación Educativa.

- Las convocatorias de ayudas

- Los espacios transversales soportados en las TIC

- La formación y las buenas prácticas

1. La Universidad Politécnica de Madrid ha optado como línea central de actuación para promover la renovación pedagógica, por la promoción y posterior reconocimiento de "Grupos de Innovación Educativa", lo cual constituye una apuesta original en el actual panorama universitario. El 27 de abril de 2005 el Consejo de Gobierno de la UPM aprobó la "Normativa de Reconocimiento de Grupos de Innovación Educativa de la Universidad Politécnica de Madrid". Esta normativa, inspirada en la ya existente de "Grupos de Investigación", tuvo un largo, participativo y laborioso proceso de elaboración dada la carencia de experiencias similares.

A través de esta iniciativa se pretende que los esfuerzos en medios y recursos con los que la UPM promueve la Innovación Educativa, reviertan en un trabajo continuo, favoreciendo su realización por grupos estables y cuya actuación tenga un mayor reconocimiento y proyección internos y externos.

Los Grupos de Innovación Educativa de la Universidad Politécnica de Madrid centran sus líneas de actividad en alguna 0 algunas de las siguientes: a) Desarrollo de nuevas Metodologías de Aprendizaje/ Evaluación.

- Metodologías activas de Aprendizaje basadas en el trabajo en grupo (Aprendizaje Cooperativo y Aprendizaje basado en Problemas/Proyectos interdisciplinares).

- Evaluación de objetivos competenciales.

-Evaluación formativa.

b) Atención al estudiante.

- Adaptación a la Universidad de los alumnos de nuevo ingreso.

- Mejora de las actividades de tutoría.

- Orientación para el empleo.

c) Desarrollo curricular.

- Diseño instructivo y planificación del aprendizaje y evaluación de asignaturas.

- Desarrollo de relaciones interdisciplinares entre asignaturas.

- Desarrollo de competencias y propuestas en torno al "Aprendizaje a lo largo de la vida".

d) Incorporación de nuevas tecnologias a la formación presencial.

- Generación de contenidos didácticos para la Red.

- Gestión del conocimiento mediada por Internet.

- Espacios virtuales de trabajo en grupo.

- Laboratorios remotos.

e) Coordinación de los programas formativos universitarios y de bachillerato

- Organización y desarrollo de acciones formativas para profesores de enseñanza secundaria.

- Elaboración de recursos didácticos compartidos.

- Participación en proyectos comunes.

f) Desarrollo de la dimensión docente en los Proyectos de $I+D+i$.

- Transferencia de conocimientos e Integración de la actividad docente e investigadora. 
- Vinculación del entorno industrial con la actividad docente.

- Análisis y Estudios: Planificación y desarrollo de estudios empíricos que permitan conocer con mayor objetividad y precisión los factores que influyen en la mejora de la calidad del programa formativo.

- Desarrollo de modelos para la evaluación de la calidad de los procesos formativos.

Para los Grupos de Innovación Educativa que sean suficientemente representativos a nivel de titulación y tengan expresamente el apoyo institucional de los centros correspondientes, se proponen, además, otras líneas de actuación más globales tales como:

- Adaptación de la organización docente a los objetivos definidos para el EEES.

- Captación de alumnos de nuevo ingreso. Fidelización de los antiguos alumnos.

- Acuerdos Internacionales con Centros de Enseñanza Superior.

En la actualidad son cerca setecientos profesores de la UPM los que están vinculados a 85 Grupos de Innovación Educativa ya formalizados en la UPM. Resulta significativa la distribución de las áreas de actividad a la que se han adscrito los mencionados grupos. El Gráfico 3 resume su distribución.
Para más información sobre los grupos de innovación educativa y su actividad se puede consultar el portal: http:// innovacioneducativa.upm.es/presentacion.html

2. Las Convocatorias de Ayudas en la UPM han sido otro instrumento muy importante en el impulso a la innovación educativa ya que se ha invertido una media de un millón de euros anuales, durante los últimos cuatro años, para la promoción de proyectos de mejora de la actividad docente. Esta inversión ha estado distribuida en diferentes convocatorias:

- Convocatoria de ayudas a la Innovación Educativa en el marco del proceso de implantación del Espacio Europeo de Educación Superior y la Mejora de la Calidad de la Enseñanza. Dicha convocatoria ha sido la más importante de todas ellas, tanto por dotación económica como por participación y resultados recogidos. Anualmente se convocan los proyectos de innovación educativa y se señalan las líneas de acción prioritarias para su desarrollo y en ellos participan cerca de 700 profesores con una tasa de aproximadamente el $60 \%$ de proyectos aceptados respecto a los presentados.

- Convocatoria de ayudas para la realización de Acciones de Movilidad vinculadas a la Innovación Educativa en el marco del proceso de implantación del Espacio Europeo de Educación Superior y la mejora de la Calidad de la

Gráfico 3. Distribución de las línea específicas de actuación seleccionadas por los Grupos de Innovación Educativa.

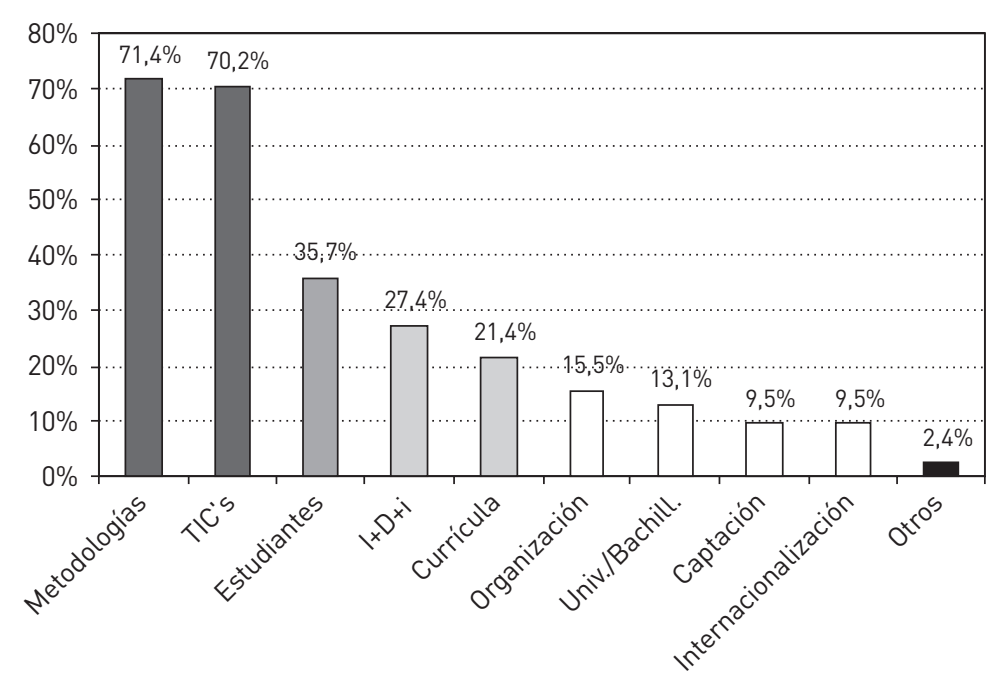


Enseñanza. Con estas convocatorias se ha pretendido apoyar aquellas iniciativas que faciliten el conocimiento e implantación de metodologías educativas que se desarrollan en otros paises integrados en el Espacio Europeo de Educación Superior. Están abiertas, en diferentes convocatorias, para el profesorado y personal de administración y servicios.

- Convocatoria de tres Premios a los Grupos de Innovación Educativa, de dos Premios a la Excelencia Docente y de diez Premios a la Innovación Educativa. Dotado cada uno con $7.500,10.000$ y 3.000 euros, respectivamente, pretende reconocer las aportaciones singulares tanto de los Grupos de Innovación educativa como a título individual.

- Convocatoria de ayudas para la impartición de asignaturas en lenguas no españolas, en las modalidades diferenciadas orientadas a profesores de instituciones extranjeras de educación superior así como en la orientada a profesores de la propia universidad. En ellas se aprovecha la flexibilidad de los créditos de libre configuración y se ofrecen cursos acreditados y que son impartidos ya sea por profesores extranjeros como por profesorado de la propia universidad en el marco de programas internacionales para el fomento de la docencia en lengua inglesa (como por ejemplo, el programa ATHENS).

- Convocatoria para la publicación de asignaturas en OpenCourseWare (OCW). A través de este proyecto, cuyo objetivo último es publicar en abierto los contenidos docentes que los profesores utilizan en sus clases, se genera un compromiso del profesor y la institución con la calidad de las asignaturas publicadas. La Universidad Politécnica de Madrid fue pionera en participar e impulsar este proyecto en nuestro país, del cual ya forman parte más de cuarenta universidades españolas.

\section{Los espacios transversales soportados en las TIC.}

La Universidad Politécnica de Madrid ha encontrado en el uso de Internet una interesante posibilidad de promover la innovación educativa compartiendo recursos y creando espacios para completar la acción en las aulas. Dos han sido las principales iniciativas: Punto de Inicio y Puesta a Punto.
Punto de Inicio se abrió en el Curso 2005-2006 y un espacio web basado en Moodle (plataforma de teleenseñanza) que ofrece material docente de autoestudio y autoevaluación (preguntas con realimentación y corrección automática) a los alumnos que ingresan en la UPM. No pretende adelantar nuevos conocimientos sino reforzar los que debe haber adquirido en el bachillerato. El material docente que se incluye es elaborado por profesores de la Universidad (grupos de profesores interdepartamentales) o tomados del material que tiene el Ministerio en el programa CNICE.

Desde el servicio de Innovación educativa se ofrecen a los Centros seis aulas diferentes: Matemáticas, Física, Dibujo, Química, Inglés Técnico y Planificación y Gestión del tiempo con una oferta de recursos didácticos de autoestudio y autoevaluación. De acuerdo al perfil de entrada, los Centros seleccionan las aulas de su interés y las completan con los recursos que consideran adecuados. El Gráfico 4 muestra un ejemplo de una de estas aulas.

Los nuevos alumnos reciben en el momento de matricularse las instrucciones procedimentales que les permiten acceder a las aulas de Punto de Inicio que están particularizadas a la titulación en la que comienzan sus estudios, manteniéndose abiertos estos espacios de trabajo de forma exclusiva para los nuevos estudiantes hasta el inicio de las actividad presencia reglada en la titulación. Posteriormente, las aulas de Punto de Inicio permanecen abiertas para todos los estudiantes de la titulación. El éxito de esta iniciativa se puede cuantificar señalando que en los meses de agosto y septiembre de 2008 , se contabilizaron 122.000 accesos y habiéndose realizado en torno a 10.000 cuestionarios de autoevaluación con una participación en todas las aulas superior al 30\%.

Esta iniciativa se desarrolla de forma complementaria y coordinada con los tradicionales "cursos cero" y a un programa de Mentorías destinado a la acogida de nuevos estudiantes.

La iniciativa Puesta a Punto tiene como objetivo el refuerzo de la formación del estudiante en la adquisición de competencias y el desarrollo de determinadas capacidades así como facilitar la acreditación de las mismas centrándose en aquellos aspectos que ha de adquirir un titulado universitario con independencia de la titulación que curse, es decir, en las competencias transversales. 
Gráfico 4. Punto de inicio: Aula de Matemáticas de la EUIT de Telecomunicación.

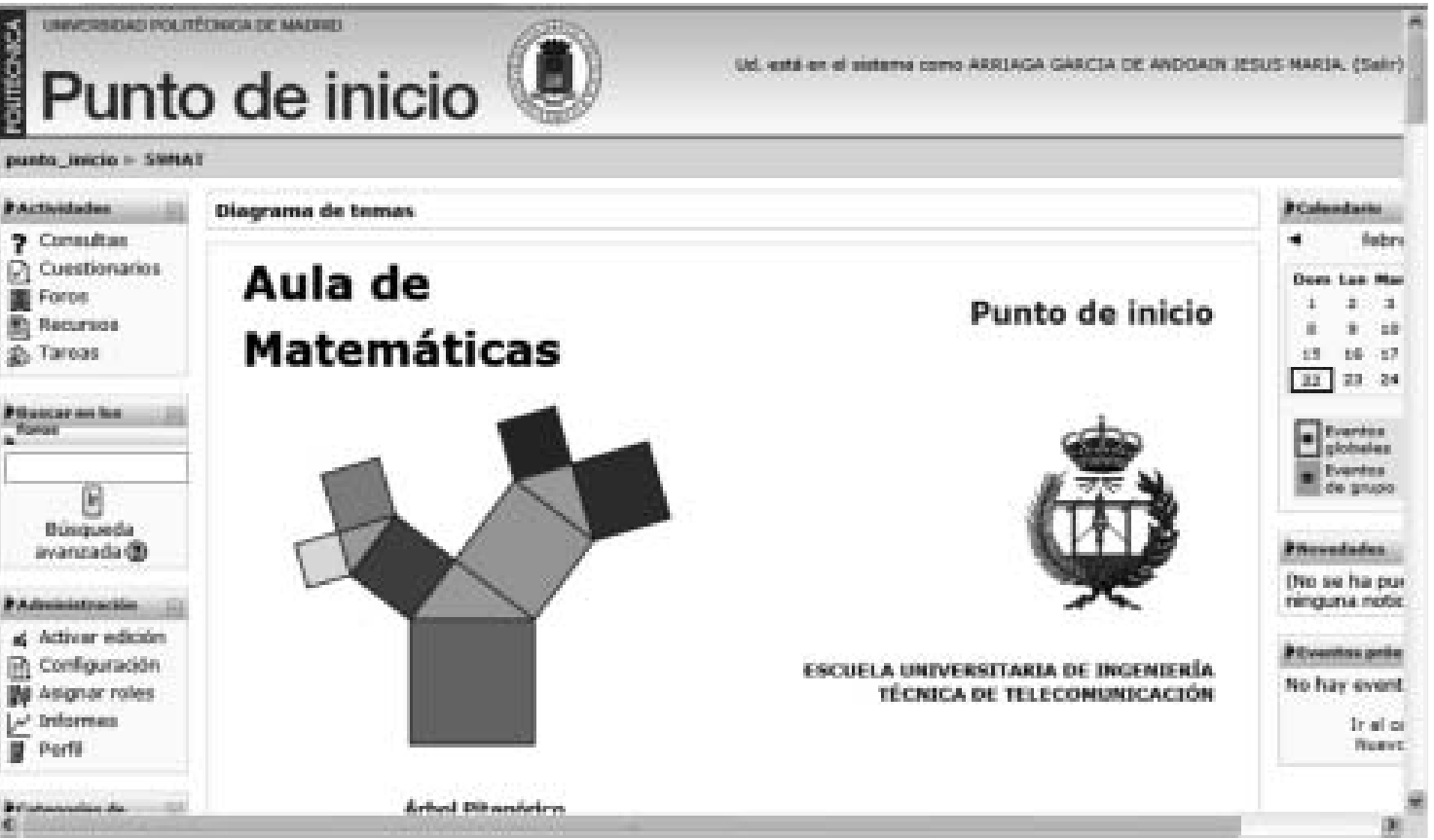

Punto de

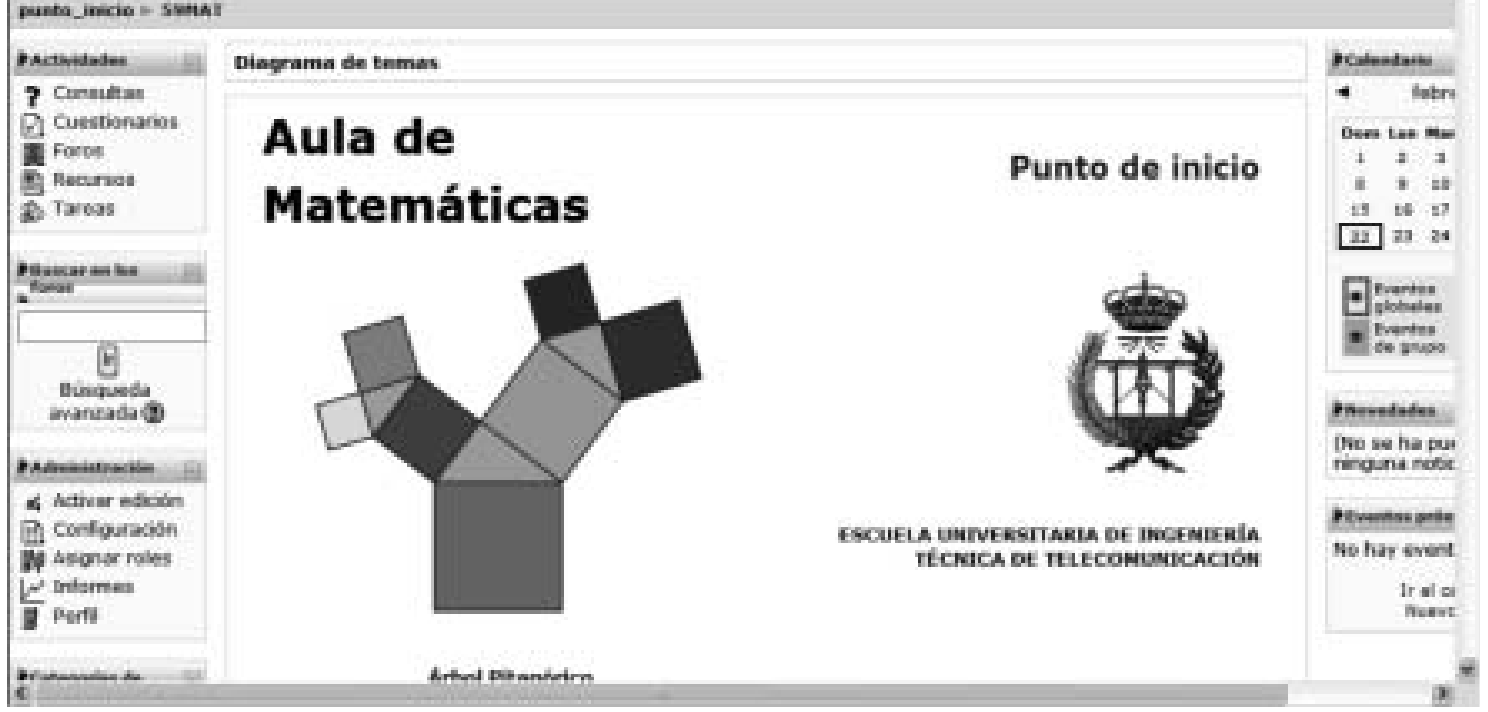
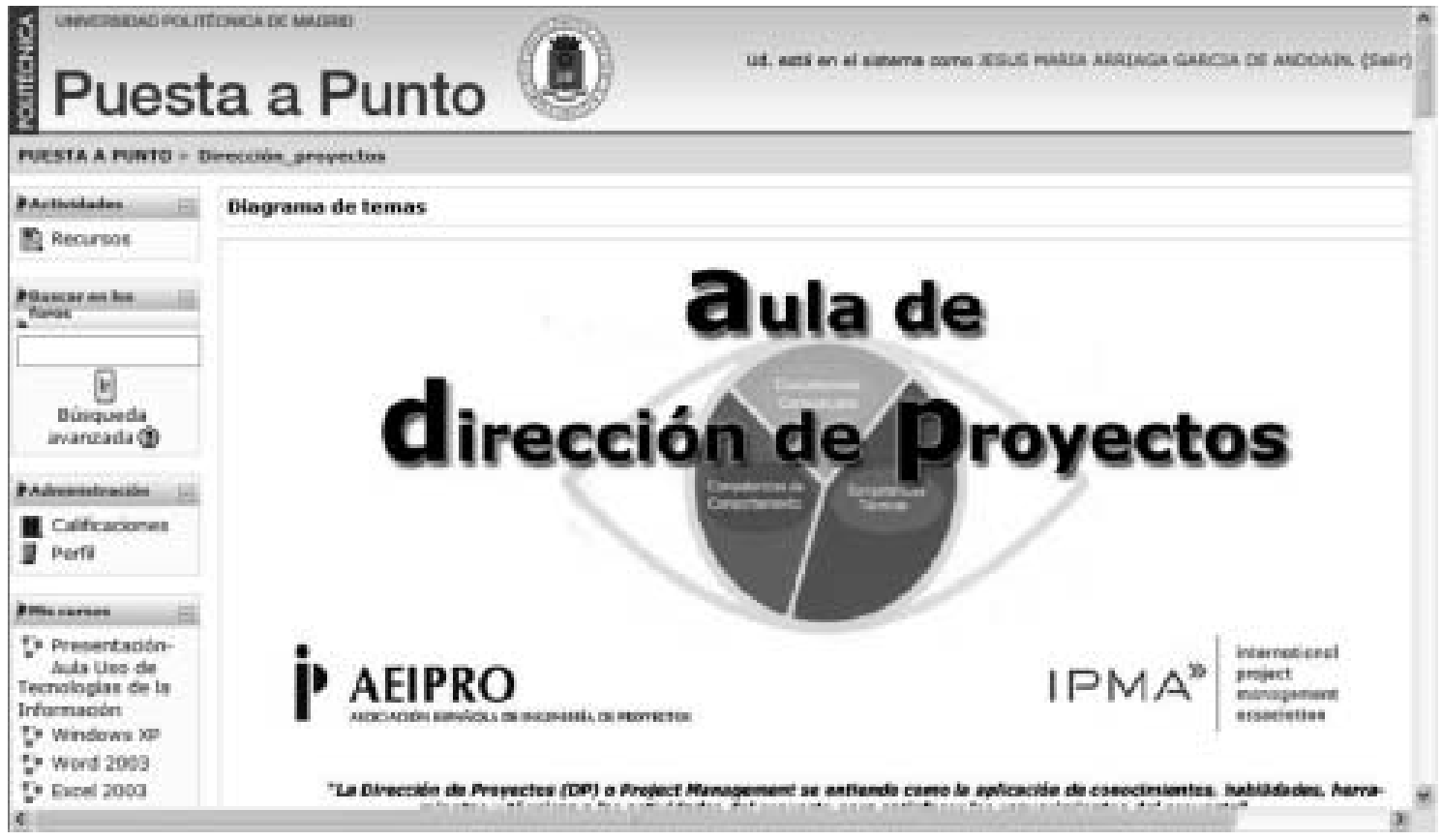
Abierta la plataforma Puesta a Punto en octubre de 2008, tiene una estructura similar al portal de Punto de Inicio, con la diferencia que esta plataforma está abierta permanentemente y a ella tienen acceso todos los alumnos matriculados en cualquier curso y titulación de la Universidad Politécnica de Madrid, así como a su profesorado y personal de administración y servicio. El Gráfico 5 muestra una vista del aula de Dirección de Proyectos. Actualmente hay abiertas las siguientes aulas:

- Tecnología de la información

- Lenguas Extranjeras (Certificación TOEIC)

- Dirección de proyectos (Certificación AEIPRO: Técnico en dirección de proyectos)

- Información para el conocimiento

- Competencias personales y participativas

- Cómo preparar una presentación en público

- Cómo hacer una buena preparación de un examen

- Como organizar tu tiempo y aprovechar las clases

- Técnicas de estudio

\section{Formación y buenas experiencias.}

Los cambios asociados a los nuevos planes de estudio sólo pueden ser abordados si están soportados en una importante sustitución de métodos clásicos por nuevas técnicas que necesitan del manejo de nuevos recursos. Por ello, el esfuerzo realizado en formación del profesorado también está siendo muy importante en los últimos años en todas las universidades españolas.

Se persigue una formación formal, al estilo de los tradicionales cursos impartidos por los "Institutos de Ciencias de la Educación" o similares, pero también se busca una formación estructurada en talleres, con programas muy centrados en temas específicos y a los que asistan colectivos de profesores que compartan proyectos comunes.

En una primera fase de este proceso de reforma tuvieron especial importancia los primeros tipos de cursos, con contenidos amplios y variados y orientados preferentemente a las competencias transversales, sistemas de tutorias, metodologías activas y todos los temas referentes a la elaboración de la memoria para la solicitud de verificación de títulos oficiales (grado y máster). Las Universidades, por lo general hemos elaborado nuestras propias guias de chequeo y un conjunto de directrices y recomendaciones que también fueron motivo de estos cursos.

En una segunda fase, en aquellos Centros que ya han presentado sus memorias para la verificación y tienen a corto plazo el inicio de los nuevos cursos, están resultando de más interés los talleres impartidos en los propios Centros, acercando la formación a los profesores con el fin de incrementar su asistencia y que la reciban grupos de profesores que comparten el proyecto común de un Programa formativo del cual todos se deben sentir corresponsables. Los contenidos en estos casos son más específicos y orientados a temas tales como:

- Formulación de objetivos, resultados de aprendizaje e indicadores.

- Aprendizaje cooperativo.

- La evaluación de competencias.

- Elaboración de guías de aprendizaje.

- Aprendizaje basado en proyectos.

- Formación interdisciplinar.

Estos talleres deben llegar también a profesores que no son muy receptivos a los cambios que se proponen, por lo que es muy aconsejable que sean impartidos por profesores de áreas del conocimiento afines y que tengan experiencia práctica de haber aplicado en su Universidad y en un contexto similar los contenidos del taller.

De forma complementaria a los cursos y talleres, han cobrado especial importancia en los últimos años la celebración de Congresos, Jornadas y otro tipo de encuentros en los que se comparten buenas prácticas. A diferencia de hace unos años en el que el número de eventos de este tipo era muy escaso y centrado en determinadas áreas del conocimiento, ahora podemos decir que nos hemos pasado al otro extremo y son "excesivos" los encuentros en torno a la innovación educativa.

Particularmente, en la Universidad Politécnica de Madrid organizamos anualmente tres tipos de Jornadas:

a) Jornadas de Intercambio de Experiencias en Innovación Educativa en la UPM. Estas Jornadas se celebran habitualmente en el mes de septiembre, son de carácter interno y en ellas se recogen las buenas prácticas 
realizadas por los profesores, particularmente como resultado de los proyectos de Innovación Educativa subvencionados por la propia UPM.

b) Jornadas Internacionales UPM sobre innovación Educativa y Convergencia Europea (INECE). Son celebradas en diciembre $y_{1}$ como su nombre indica, están abiertas a profesores de otras universidades nacionales $y$ extranjeras. Aunque mantiene un programa centrado en las comunicaciones seleccionadas por el Comité Científico, estas Jornadas presentan una interesante oferta de conferencias invitadas

c) Educación Científico-Tecnológica (ECITEC). Con estas Jornadas se intenta adquirir un mayor conocimiento sobre los problemas de acceso e integración de los alumnos a los estudios técnicos universitarios y elaborar propuestas de mejora que puedan ser desarrolladas por la Universidad Politécnica de Madrid en colaboración con los Centros de Enseñanzas Medias y la Consejería de Educación de la Comunidad de Madrid. Son convocadas conjuntamente por la Universidad Politécnica y la Consejería de Educación de la Comunidad de Madrid.

\section{Conclusiones}

La integración en el Espacio Europeo de Educación Superior de las universidades españolas tendrá como consecuencia más significativa la renovación de los métodos docentes tradicionalmente utilizados, incorporando, junto a algunos métodos clásicos, nuevos métodos que impliquen una mayor participación de los estudiantes en su proceso formativo. Sólo de esta forma se podrá ofrecer a los estudiantes una formación que, manteniendo el nivel con el que ahora adquieren competencias especificas de su titulación, incorpore la adquisición de competencias transversales altamente demandadas por el entorno social y profesional.

Pero para que los procesos de innovación educativa puedan dar sus frutos es necesario que los nuevos planes de estudio se desarrollen de una forma racional, con una visión de conjunto de todos los elementos que el marco legislativo español identifica en los planes de estudios de los nuevos títulos oficiales universitarios.

Junto a ello, las universidades españolas deben continuar los esfuerzos que desde hace unos años han venido desarrollando en la formación pedagógica de sus docentes, implantando planes de formación del profesorado y diseñando estrategias de apoyo y reconocimiento de esta actividad.

El análisis de las primeras experiencias realizadas en esta dirección en la Universidad Politécnica de Madrid ofrece resultados esperanzadores pero que necesitarán ser continuados y reforzados para implicar en ellos a un mayor número de docentes.

Es especialmente necesario en estos momentos de implantación de los nuevos planes de estudios que las autoridades gubernativas sea sensibles a este proceso de cambio, tomando consciencia de que la autentica reforma no está tanto en la estructura de los títulos oficiales como en la mejora de los procesos de aprendizaje, y previendo los apoyos necesarios para que la reforma pueda realizarse con las mejores garantías de éxito.

Recibido: 16 de marzo de 2009

Aceptado: 16 de abril de 2009 